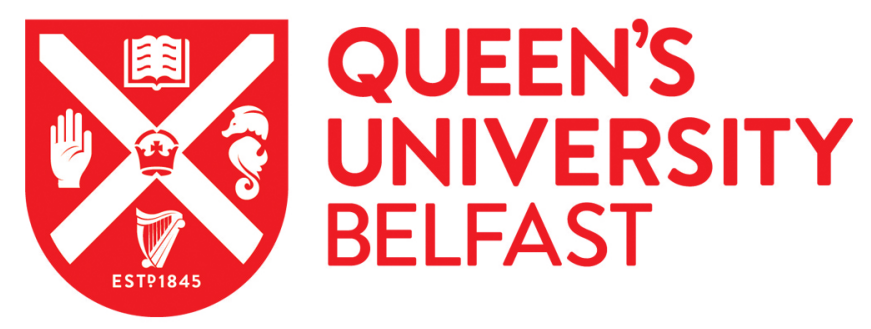

\title{
The algebraic K-theory of the projective line associated with a strongly Z-graded ring
}

Hüttemann, T., \& Montgomery, T. (2020). The algebraic K-theory of the projective line associated with a strongly Z-graded ring. Journal of Pure and Applied Algebra, 224(12), [106425].

https://doi.org/10.1016/j.jpaa.2020.106425

Published in:

Journal of Pure and Applied Algebra

Document Version:

Peer reviewed version

Queen's University Belfast - Research Portal:

Link to publication record in Queen's University Belfast Research Portal

Publisher rights

(c) 2020 Elsevier B.V. All rights reserved.

This manuscript is distributed under a Creative Commons Attribution-NonCommercial-NoDerivs License

(https://creativecommons.org/licenses/by-nc-nd/4.0/), which permits distribution and reproduction for non-commercial purposes, provided the author and source are cited.

\section{General rights}

Copyright for the publications made accessible via the Queen's University Belfast Research Portal is retained by the author(s) and / or other copyright owners and it is a condition of accessing these publications that users recognise and abide by the legal requirements associated with these rights.

Take down policy

The Research Portal is Queen's institutional repository that provides access to Queen's research output. Every effort has been made to ensure that content in the Research Portal does not infringe any person's rights, or applicable UK laws. If you discover content in the Research Portal that you believe breaches copyright or violates any law, please contact openaccess@qub.ac.uk. 


\title{
THE ALGEBRAIC $K$-THEORY OF THE PROJECTIVE LINE ASSOCIATED WITH A STRONGLY $\mathbb{Z}$-GRADED RING
}

\author{
THOMAS HÜTTEMANN AND TASHA MONTGOMERY
}

\begin{abstract}
A LAURENT polynomial ring $R_{0}\left[t, t^{-1}\right]$ with coefficients in a unital ring determines a category of quasi-coherent sheaves on the projective line over $R_{0}$; its $K$-theory is known to split into a direct sum of two copies of the $K$-theory of $R_{0}$. In this paper, the result is generalised to the case of an arbitrary strongly $\mathbb{Z}$-graded ring $R$ in place of the LAURENT polynomial ring. The projective line associated with $R$ is indirectly defined by specifying the corresponding category of quasi-coherent sheaves. Notions from algebraic geometry like sheaf cohomology and twisting sheaves are transferred to the new setting, and the $K$-theoretical splitting is established.
\end{abstract}

\section{CONTENTS}

Introduction

- The projective line and its algebraic $K$-theory

- Acknowledgements

Part I. On strongly $\mathbb{Z}$-graded rings

I.1. Conventions and notation used in the paper 3

I.2. $\quad$ Strongly graded rings

Part II. The projective line of a strongly $\mathbb{Z}$-graded ring 6

II.1. Sheaves on the projective line 6

\begin{tabular}{lll}
\hline II.2. & Vector bundles and twisting sheaves & 7
\end{tabular}

II.3. Sheaf cohomology 9

II.4. Vector bundles with trivial first cohomology $\quad 11$

Part III. The algebraic $K$-theory of the projective line 14

$\begin{array}{lll}\text { III.1. Algebraic } K \text {-theory } & 14\end{array}$

\begin{tabular}{lll}
\hline III.2. Canonical sheaves and global sections & 15
\end{tabular}

\begin{tabular}{lll}
\hline III.3. Weak equivalences of complexes of sheaves & 18
\end{tabular}

\begin{tabular}{lll}
\hline III.4. Acyclic complexes of vector bundles & 19
\end{tabular}

$\begin{array}{lll}\text { III.5. The splitting result } & 21\end{array}$

\begin{tabular}{ll}
\hline References & 23
\end{tabular}

Date: April 27, 2020.

2010 Mathematics Subject Classification. Primary 19D55; Secondary 16W50, 19 E99. 


\section{Introduction}

The projective line and its algebraic $K$-theory. The projective line $\mathbb{P}^{1}=\mathbb{P}_{R_{0}}^{1}$ over a commutative unital ring $R_{0}$ is, by definition, the scheme Proj $R_{0}[x, y]$; it is covered by ZARISKI open subsets $\operatorname{Spec} R_{0}[t]$ and $\operatorname{Spec} R_{0}\left[t^{-1}\right]$ with intersection $\operatorname{Spec} R_{0}\left[t, t^{-1}\right]$, where $t=x y^{-1}$. The category of quasi-coherent sheaves of $\mathscr{O}_{\mathbb{P}^{1}}$-modules on the projective line is equivalent to a category of diagrams of the form

$$
M^{-} \longrightarrow M^{0} \longleftarrow M^{+}
$$

with entries modules over the rings $R_{0}\left[t^{-1}\right], R_{0}\left[t, t^{-1}\right]$ and $R_{0}[t]$, respectively, subject to a certain gluing condition. Even when $R_{0}$ fails to be commutative we can define a quasi-coherent sheaf on the projective line to be such a diagram. This generalisation has been considered in the context of algebraic $K$-theory by BASS [Bas68, XXII.9] and QUILLEN [Qui73, \$8], who showed that there is a splitting of QUILLEN $K$-groups

$$
K_{q}\left(\mathbb{P}^{1}\right) \cong K_{q}\left(R_{0}\right) \oplus K_{q}\left(R_{0}\right)
$$

for all $q \geq 0$, where the left-hand side denotes the $K$-groups of a category of diagrams as above subject to suitable finiteness conditions. One can even go further and replace the (LAURENT) polynomial rings by twisted (LAURENT) polynomial rings with respect to an automorphism of $R_{0}$. This has been observed by GRAYSON [Gra88, Theorem 1.1], and an explicit proof containing full technical details has been made available by YAO [Yao95, $\$ 1]$.

A more drastic generalisation may be attempted, starting from the observation that the LAURENT polynomial ring $R_{0}\left[t, t^{-1}\right]$ is a $\mathbb{Z}$-graded ring, and that the polynomial rings $R_{0}\left[t^{-1}\right]$ and $R_{0}[t]$ are the subrings generated by elements of non-positive resp. non-negative degrees. It will be shown in this paper that the $K$-theoretical splitting result above is still valid in this generalised situation, provided the $\mathbb{Z}$-graded ring in question is in fact strongly $\mathbb{Z}$-graded.

The splitting result is a key ingredient for generalising the fundamental theorem algebraic $K$-theory from LAURENT polynomial rings to strongly $\mathbb{Z}$-graded rings (HÜTTEMANN [Hüt20]). The theorem relates the algebraic $K$-groups of the ring $R=\bigoplus_{k \in \mathbb{Z}} R_{k}$ with subgroups and quotients of the algebraic $K$-groups of $R_{0}$ determined by the graded structure. The projective line has also been used by STEERS and the first author to promote results on algebraic finite domination of chain complexes from LAURENT polynomial rings to the class of strongly $\mathbb{Z}$-graded rings [HS17].

Strong gradings were introduced by DADE [Dad80] to capture and generalise the quintessential properties of group rings; as in the original representationtheoretic applications, it is the strongly graded structure rather than the special form of group rings that controls the splitting behaviour of algebraic $K$-theory. 
It might be worthwhile to illustrate the gain in generality by an explicit example. For $K$ a field the (commutative) ring

$$
R=K[A, B, C, D] /(A B+C D-1),
$$

graded by $\operatorname{deg}(A)=\operatorname{deg}(C)=1$ and $\operatorname{deg}(B)=\operatorname{deg}(D)=-1$, is a strongly $\mathbb{Z}$-graded ring; it can be shown that $R$ contains only the trivial units $K^{\times} \subset R$ so that $R$ is not a crossed product, and certainly not a (twisted) LAURENT polynomial ring. Other examples of strongly $\mathbb{Z}$-graded rings include LEAVITT algebras of type $(1, n)$, or more generally LEAVITT path algebras associated with finite graphs without sink (HAZRAT [Haz13, Theorem 3.15]). The splitting result (†) remains valid for the projective lines associated with these rings.

The proof follows broadly the pattern laid out by QUILLEN [Qui73, \$8], formulated in terms of WALDHAUSEN's $\mathscr{S}$-construction [Wal85]. One difference to note is that we eschew the use of regular sheaves in favour of a simpler notion of vector bundles without higher sheaf cohomology. Moreover, there are major deviations from the template in several places necessitated by the much larger class of rings under consideration. The increased generality leads to unexpected phenomena. For example, we encounter a double-indexed family of non-isomorphic twisting sheaves $\mathscr{O}(k, \ell)$ on the projective line in place of the more familiar singleindexed family $\mathscr{O}(k+\ell)$; the resulting twist operation leads, in effect, to a $\mathbb{Z}$-action on the collection of cohomology modules of a given sheaf (Theorem II.3.5).

Compared to the existing treatments of twisted LAURENT polynomial rings the graded techniques are simple and elegant, avoiding all unpleasantries with changing module structures by the prescribed ring automorphism. This indicates that strongly graded rings are the "right" generalisation of groups rings, even in contexts not originally considered by DADE.

Acknowledgements. This paper is part of the second author's $\mathrm{PhD}$ thesis. The idea to consider the projective space associated with a strongly $\mathbb{Z}$-graded ring was developed during a research visit of the first author to Beijing Institute of Technology. Their hospitality and financial support are greatly appreciated.

\section{Part I. On strongly $\mathbb{Z}$-graded rings}

\section{I.1. CONVENTIONS AND NOTATION USED IN THE PAPER}

A "ring" will always mean an associative unital ring. The term "module", if unspecified, always refers to a unital right module over the ring in question.

Let $R=\bigoplus_{\mathbb{Z}} R_{k}$ be a unital $\mathbb{Z}$-graded ring. As usual, the elements of $R_{k}$ are called the homogeneous elements of degree $k$. It is known that $R_{0}$ is a subring of $R$ with the same unit element as $R$ [Dad80, Proposition 1.4]. The two sets

$$
R_{\leq 0}=\bigoplus_{n \leq 0} R_{n} \quad \text { and } \quad R_{\geq 0}=\bigoplus_{n \geq 0} R_{n}
$$


are $\mathbb{Z}$-graded subrings of $R$. We write $R_{\leq k}$ for the $R_{\leq 0}$-module $\bigoplus_{n \leq k} R_{n}$, and $R_{\geq-\ell}$ for the $R_{\geq 0}$-module $\bigoplus_{n \geq-\ell} R_{n}$.

The reader may want to keep the following motivating example in mind: $R_{0}$ a commutative unital ring, $R=R_{0}\left[t, t^{-1}\right]$ the LAURENT polynomial ring in one indeterminate, $R_{\leq 0}=R_{0}\left[t^{-1}\right]$ and $R_{\geq 0}=R_{0}[t]$ the polynomial rings. Then $R_{\geq-\ell}=t^{-\ell} R_{0}[t]$ and $R_{\leq k}=t^{k} R_{0}\left[t^{-1}\right]$. - A more complicated example, requiring the full force of strongly graded techniques to be introduced presently, is the graded ring $R=K[A, B, C, D] /(A B+C D-1)$ introduced in ( $\ddagger$ ) above.

\section{I.2. STRONGLY GRADED RINGS}

Let $R=\bigoplus_{k \in \mathbb{Z}} R_{k}$ be a $\mathbb{Z}$-graded ring. A partition of unity of type $(k,-k)$ is a finite sum expression $1=\sum_{j} \lambda_{j} e_{j}$ with $\lambda_{j} \in R_{k}$ and $e_{j} \in R_{-k}$. Partitions of unity may or may not exist in general. - Following DADE [Dad80] we call $R$ a strongly $\mathbb{Z}$-graded ring if $R_{k} R_{\ell}=R_{k+\ell}$ for all $k, \ell \in \mathbb{Z}$, where the left-hand side consists of all finite sums of products $a b$ with $a \in R_{k}$ and $b \in R_{\ell}$.

Proposition I.2.1 (Characterisation of strongly graded rings; see DADE [Dad80, Proposition 1.6], and also HÜTTEMANN and STEERS [HS17, Proposition 1.5]). The following statements are equivalent:

(1) The $\mathbb{Z}$-graded ring $R$ is strongly graded.

(2) For every $k \in \mathbb{Z}$ there is at least one partition of unity of type $(k,-k)$.

(3) There is at least one partition of unity of type $(1,-1)$, and at least one partition of unity of type $(-1,1)$.

We now discuss the basic properties of strongly graded rings. Though most are well known, we include some proofs to emphasise the benefits of strong grading.

Lemma I.2.2. Let $R$ be a strongly $\mathbb{Z}$-graded ring, and let $n \in \mathbb{Z}$. The homogeneous component $R_{n}$ is a finitely generated projective $R_{0}$-module. The $R_{\geq 0}$-module $R_{\geq-n}$ is finitely generated projective, as is the $R_{\leq 0}$-module $R_{\leq n}$. These statements are true for both left and right module versions.

Proof. We will deal with the second statement (right module version only); the first part has an almost identical proof, the third part is true by symmetry. Let $1=$ $\sum_{j} \lambda_{j} \varrho_{j}$ be a partition of unity of type $(-n, n)$. Then we have right $R_{\geq 0}$-linear maps

$$
\beta_{j}: R_{\geq-n} \longrightarrow R_{\geq 0}, \quad x \mapsto e_{j} x
$$

such that $\sum_{j} \lambda_{j} \beta_{j}$ is the identity map of $R_{\geq-n}$. By the dual basis theorem [Bou98, $\$$ III.2 No. 6, Proposition 12] this means that $R_{\geq-n}$ is a finitely generated projective $R_{\geq 0}$-module (with generators $\lambda_{j}$ ).

Corollary I.2.3. Suppose that $R$ is a strongly $\mathbb{Z}$-graded ring. Then $R$ is a flat left $R_{\geq 0}$-module, and a flat left $R_{\leq 0}$-module. 
Proof. The left $R_{\geq 0}$-modules $R_{\geq-n}$ are projective, by Lemma I.2.2, hence their nested union $\bigcup_{n \geq 0} R_{\geq-n}=R$ is flat. The other statement has a symmetric proof.

Lemma I.2.4. Let $R$ be a strongly $\mathbb{Z}$-graded ring, and let $k, \ell \in \mathbb{Z}$. The multiplication map $\mu: a \otimes b \mapsto a b$ yields isomorphisms

$$
\begin{aligned}
R_{\leq \ell} \otimes_{R_{0}} R_{k} \cong R_{\leq \ell+k} & & \text { (as } R_{\leq 0}-R_{0} \text {-bimodules), } \\
R_{\leq k} \otimes_{R_{\leq 0}} R \cong R & & \text { (as } R_{\leq 0}-R \text {-bimodules), } \\
R_{\leq \ell} \otimes_{R_{\leq 0}} R_{\leq k} \cong R_{\leq \ell+k} & & \text { (as } R_{\leq 0}-R_{\leq 0} \text {-bimodules), } \\
R_{k} \otimes_{R_{0}} R_{\ell} \cong R_{k+\ell} & & \text { (as } R_{0}-R_{0} \text {-bimodules), } \\
R_{\geq-k} \otimes_{R_{\geq 0}} R_{\geq-\ell} \cong R_{\geq-k-\ell} & & \text { (as } R_{\geq 0}-R_{\geq 0} \text { bimodules), } \\
R_{\geq-\ell} \otimes_{R_{\geq 0}} \cong \cong R & & \text { (as } R_{\geq 0}-R \text {-bimodules), } \\
R_{\geq-k} \otimes_{R_{0}} R_{-\ell} \cong R_{\geq-k-\ell} & & \text { (as } R_{\geq 0}-R_{0} \text {-bimodules). }
\end{aligned}
$$

In particular, $R_{\leq k}$ is an invertible $R_{\leq 0}$-bimodule with inverse $R_{\leq-k}$ by (I.2.7), $R_{k}$ is an invertible $R_{0}$-bimodule with inverse $R_{-k}$ by (I.2.8), and $R_{\geq-k}$ is an invertible $R_{\geq 0}$-bimodule with inverse $R_{\geq k}$ by (I.2.9).

Proof. We prove (I.2.5) only, the other statements have similar proofs. Let $1=$ $\sum_{j} x_{j} y_{j}$ be a partition of unity of type $(-k, k)$. The map

$$
\varrho: R_{\leq \ell+k} \longrightarrow R_{\leq \ell} \otimes_{R_{0}} R_{k}, \quad r \mapsto \sum_{j} r x_{j} \otimes y_{j}
$$

is an $R_{\leq 0}-R_{0}$-bimodule map; indeed, linearity on the left is immediate from the definition, while for $r \in R_{\leq \ell+k}$ and $s \in R_{0}$ we calculate (using $y_{k} s x_{j} \in R_{0}$ at the third equality sign)

$$
\begin{aligned}
\varrho(r s)=\sum_{j} r s x_{j} \otimes y_{j} & =\sum_{j} \sum_{k} r x_{k} y_{k} s x_{j} \otimes y_{j} \\
& =\sum_{k} \sum_{j} r x_{k} \otimes y_{k} s x_{j} y_{j}=\sum_{k} r x_{k} \otimes y_{k} s=\varrho(r) \cdot s .
\end{aligned}
$$

We have $\mu \varrho(r)=\sum_{j} r x_{j} y_{j}=r$, and also, for $r \in R_{\leq \ell}$ and $s \in R_{k}$,

$$
e \mu(r \otimes s)=e(r s)=\sum_{j} r s x_{j} \otimes y_{j}=\sum_{j} r \otimes s x_{j} y_{j}=r \otimes s
$$

so that $\mu$ is an isomorphism with inverse $e$.

Proposition I.2.12. Let $R$ be a strongly $\mathbb{Z}$-graded ring, let $M$ be an $R_{\leq 0}$-module, and let $k \in \mathbb{Z}$. The map

$$
\Omega_{M}: M \otimes_{R_{0}} R_{k} \longrightarrow M \otimes_{R_{\leq 0}} R_{\leq k}, \quad x \otimes y \mapsto x \otimes y
$$


of right $R_{0}$-modules is an isomorphism. Similarly, there are isomorphisms of right $R_{0}$-modules $N \otimes_{R_{0}} R_{-\ell} \longrightarrow N \otimes_{R_{\geq 0}} R_{\geq-\ell}$ and $Q \otimes_{R_{0}} R_{k} \longrightarrow Q \otimes_{R} R$ for any $R_{\geq 0}$-module $N$ and any $R$-module $Q$, and any integer $\ell \in \mathbb{Z}$.

Proof. For $M=R_{\leq 0}$ this is true since both source and target are isomorphic, via the multiplication map $m \otimes r \mapsto m r$, to $R_{\leq k}$ by (I.2.5) and (I.2.7). By taking direct sums the claim follows for $M$ a free $R_{\leq 0}$-module (with a specified basis, possibly infinite). The general case follows from considering a free $R_{\leq 0}$-module presentation of $M$ : choose free modules $G$ and $F$ and maps so as to obtain an exact sequence $G \longrightarrow F \longrightarrow M \longrightarrow 0$, and produce from this a commutative diagram

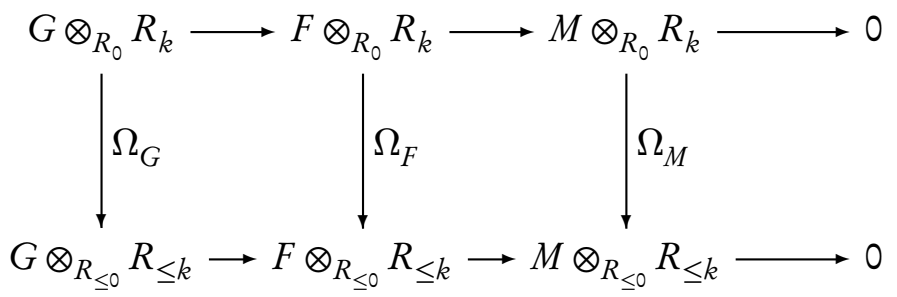

which has exact rows since tensor products are right exact. We know already that $\Omega_{G}$ and $\Omega_{F}$ are isomorphisms, hence so is $\Omega_{M}$ by the five lemma. - The remaining statements are proved in a similar fashion.

\section{Part II. The projective line of a strongly $\mathbb{Z}$-graded ring}

From now on, we assume for the remainder of the paper that $R=\bigoplus_{k \in \mathbb{Z}} R_{k}$ is a strongly $\mathbb{Z}$-graded ring.

\section{II.1. SHEAVES ON THE PROJECTIVE LINE}

Definition II.1.1. A quasi-coherent sheaf on $\mathbb{P}^{1}$, or just sheaf for short, is a diagram

$$
\mathscr{Y}=\left(Y^{-} \stackrel{u^{-}}{\longrightarrow} Y^{0} \stackrel{u^{+}}{\longrightarrow} Y^{+}\right)
$$

where $Y^{-}, Y^{0}$ and $Y^{+}$are modules over $R_{\leq 0}, R$ and $R_{\geq 0}$, respectively, with an $R_{\leq 0^{-}}$-linear homomorphisms $U^{-}$and an $R_{\geq 0}$-linear homomorphism $U^{+}$, such that the diagram of the adjoint $R$-linear maps

$$
Y^{-} \otimes_{R_{\leq 0}} R \underset{\sharp}{\stackrel{u_{\sharp}^{-}}{\cong}} Y^{0} \stackrel{u_{\sharp}^{+}}{\cong} Y^{+} \otimes_{R_{\geq 0}} R
$$

consists of isomorphisms. This latter condition will be referred to as the sheaf condition.

These quasi-coherent sheaves form a category $\mathfrak{Q} \mathfrak{C o h}\left(\mathbb{P}^{1}\right)$ in an obvious way; a morphism $f: \mathscr{Y} \longrightarrow \mathscr{Z}$ is a triple $f=\left(f^{-}, f^{0}, f^{+}\right)$of linear maps (of modules over different rings) compatible with the structure maps $u^{ \pm}$of $\mathscr{Y}$ and the structure maps $\zeta^{ \pm}$of $\mathscr{Z}$ in the sense that $f^{0} \circ u^{ \pm}=\zeta^{ \pm} \circ f^{ \pm}$. In fact, $\mathfrak{Q} \mathfrak{C o h}\left(\mathbb{P}^{1}\right)$ 
is an ABELian category. Kernels and cokernels are computed pointwise as in diagram categories; the only non-trivial thing to verify is that, given a morphism $f: \mathscr{Y} \longrightarrow \mathscr{Z}$ of sheaves, the diagram

$$
\operatorname{ker}(f)=\left(\operatorname{ker}\left(f^{-}\right) \stackrel{\varkappa^{-}}{\longrightarrow} \operatorname{ker}\left(f^{0}\right) \stackrel{\chi^{+}}{\longrightarrow} \operatorname{ker}\left(f^{+}\right)\right)
$$

is a sheaf itself. This follows from the five lemma, applied to the diagram below.

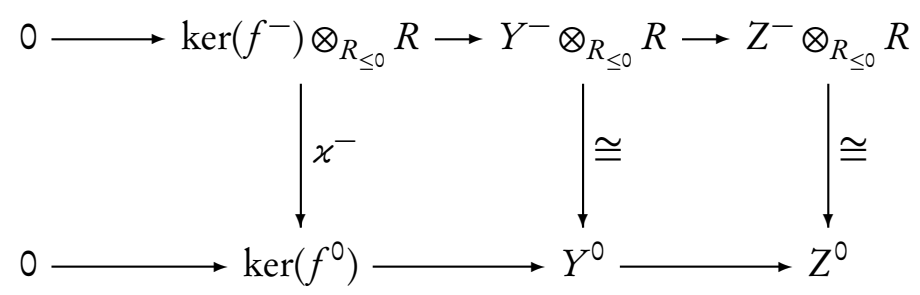

The top row is exact as $R$ is a flat left $R_{\leq 0}$-module by Corollary I.2.3; the second and third vertical maps are isomorphisms as $\mathscr{Y}$ and $\mathscr{Z}$ are sheaves. This forces $\chi^{-}$to be an isomorphism as well. The argument works mutatis mutandis for $\chi^{+}$. - Working in an ABELian category we have a canonical notion of exactness at our disposal: a sequence of sheaves and morphisms $0 \longrightarrow \mathscr{X} \stackrel{g}{\longrightarrow} \mathscr{Y} \stackrel{f}{\longrightarrow} \mathscr{Z} \longrightarrow 0$ is (short) exact if for all decorations $? \in\{-, 0,+\}$ the sequence

$$
0 \longrightarrow X^{?} \stackrel{g^{?}}{\longrightarrow} Y^{?} \stackrel{f^{?}}{\longrightarrow} Z^{?} \longrightarrow 0
$$

is an exact sequence of $R_{0}$-modules.

\section{II.2. VECTOR BUNDLES AND TWISTING SHEAVES}

Definition II.2.1. The sheaf $\mathscr{Y}$ of (II.1.2) is called locally finitely generated if its constituent modules are finitely generated as modules over their respective ground rings. We call $\mathscr{Y}$ a vector bundle if its constituent modules are finitely generated projective modules over their respective ground rings. The category of vector bundles (and all morphisms of sheaves between them) is denoted by $\operatorname{Vect}\left(\mathbb{P}^{1}\right)$.

Important examples of vector bundles are the twisting sheaves

$$
\mathscr{O}(k, \ell)=\left(R_{\leq k} \stackrel{\subseteq}{\longrightarrow} R \stackrel{\supseteq}{\longrightarrow} R_{\geq-\ell}\right)
$$

where $k$ and $\ell$ are integers. These objects are sheaves by Lemma I.2.4, and are indeed vector bundles by Lemma I.2.2. For us $\mathscr{O}(k, \ell)$ plays the rôle of what is denoted by $\mathscr{O}_{\mathbb{P}^{1}}(k+\ell)$ in algebraic geometry. It should be emphasised that in general the twisting sheaves as defined here depend on both $k$ and $\ell$ and not just on the sum $k+\ell$. If, however, $R$ is a crossed product (equivalently, if $R$ contains an invertible homogeneous element of degree 1) then the sum $k+\ell$ determines $\mathscr{O}(k, \ell)$ up to isomorphism in $\operatorname{Vect}\left(\mathbb{P}^{1}\right)$. 
Proposition II.2.2. Let $k, \ell \in \mathbb{Z}$. The commuting square of twisting sheaves and inclusion maps

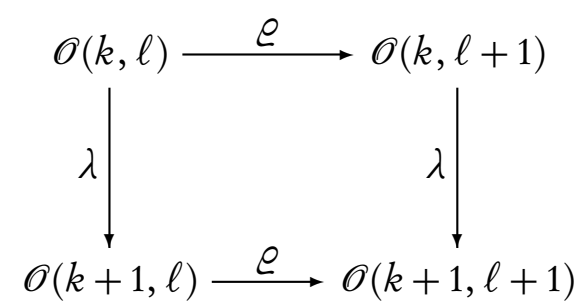

is cartesian in the sense that there results a short exact sequence of sheaves

$$
\begin{aligned}
0 \longrightarrow \mathscr{O}(k, \ell) \stackrel{\left(\begin{array}{l}
\lambda \\
\varrho
\end{array}\right)}{\longrightarrow} \mathscr{O}(k+1, \ell) & \oplus \mathscr{O}(k, \ell+1) \\
\stackrel{(-\varrho, \lambda)}{\longrightarrow} & \mathscr{O}(k+1, \ell+1) \longrightarrow 0 .
\end{aligned}
$$

Proof. Restricting to “-”-components, the sequence (II.2.3) becomes the sequence of $R_{\leq 0}$-modules

$$
0 \longrightarrow R_{\leq k} \stackrel{\left(\begin{array}{l}
1 \\
1
\end{array}\right)}{\longrightarrow} R_{\leq k+1} \oplus R_{\leq k} \stackrel{(-11)}{\longrightarrow} R_{\leq k+1} \longrightarrow 0
$$

which is clearly exact. Similarly for the other two components.

Definition II.2.4. Let $\mathscr{Y}$ be a sheaf, and let $k, \ell \in \mathbb{Z}$. We define the $(k, \ell)$ th $t w i s t$ of $\mathscr{Y}$, denoted $\mathscr{Y}(k, \ell)$, to be the sheaf

$$
\mathscr{Y}(k, \ell)=\left(Y^{-} \otimes_{R_{\leq 0}} R_{\leq k} \longrightarrow Y^{0} \otimes_{R} R \longleftarrow Y^{+} \otimes_{R_{\geq 0}} R_{\geq-\ell}\right),
$$

with structure maps induced by those of $\mathscr{Y}$ and the inclusion maps.

One should maybe think of $\mathscr{Y}(k, \ell)$ as the " $(k+\ell)$ th twist of $\mathscr{Y}$ " in the sense of algebraic geometry. - Twisting is functorial, and is in fact exact: a short exact sequence of sheaves

$$
0 \longrightarrow \mathscr{X} \longrightarrow \mathscr{Y} \longrightarrow \mathscr{Z} \longrightarrow 0
$$

is transformed into another short exact sequence

$$
0 \longrightarrow \mathscr{X}(k, \ell) \longrightarrow \mathscr{Y}(k, \ell) \longrightarrow \mathscr{Z}(k, \ell) \longrightarrow 0 .
$$

This is true as each entry in the twisted version is obtained from the untwisted one by taking the tensor product with a projective, hence flat, module over the relevant ring, cf. Lemma I.2.2. Moreover, there are isomorphisms, natural in the sheaf $\mathscr{Y}$,

$$
\mathscr{Y}(k, \ell)\left(k^{\prime}, \ell^{\prime}\right) \cong \mathscr{Y}\left(k+k^{\prime}, \ell+\ell^{\prime}\right) \text { and } \mathscr{Y}(0,0) \cong \mathscr{Y},
$$

as follows immediately from the isomorphisms listed in Lemma I.2.4.

Lemma II.2.6. Let $\mathscr{Y}$ be a locally finitely generated sheaf. Then there exists a vector bundle $\mathscr{F}$ and an epimorphism $\mathscr{F} \longrightarrow \mathscr{Y}$, where $\mathscr{F}$ is a finite direct sum of twisting sheaves $\mathscr{O}(k, \ell)$ with $k, \ell \leq 0$ and $k+\ell<0$. 
Proof. Let $y \in Y^{0}$ be an arbitrary element. As tensor products commute with nested unions, and as $\mathscr{Y}$ satisfies the sheaf condition (II.1.3), we can consider $y$ as an element in the ABELian group

$$
Y^{0} \cong Y^{+} \otimes_{R_{\geq 0}} R=Y^{+} \otimes_{R_{\geq 0}} \bigcup_{n^{+} \geq 0} R_{\geq-n^{+}}=\bigcup_{n^{+} \geq 0} Y^{+} \otimes_{R_{\geq 0}} R_{\geq-n^{+}}
$$

that is, we find $n^{+}>0$ and an element $y^{+} \in Y^{+} \otimes_{R_{\geq 0}} R_{\geq-n^{+}}$such that $y$ is the image of $y^{+}$under the map $z \otimes r \mapsto u^{+}(z) r$. Similarly, we find $n^{-}>0$ and an element $y^{-} \in Y^{-} \otimes_{R_{\leq 0}} R_{\leq n^{-}}$such that $y$ is the image of $y^{-}$under the map $z \otimes r \mapsto u^{-}(z) r$. We thus get a map of sheaves $g=\left(g^{-}, g^{0}, g^{+}\right): \mathscr{O}(0,0) \longrightarrow \mathscr{Y}\left(n^{-}, n^{+}\right)$determined by sending the elements 1 of $R_{\leq 0}, R$ and $R_{\geq 0}$ to $y^{-}, y$ and $y^{+}$, respectively. Write $k=-n^{-}<0$ and $\ell=-n^{+}<0$; by twisting we obtain a map of sheaves

$$
g(k, \ell)=f=\left(f^{-}, f^{0}, f^{+}\right): \mathscr{O}(k, \ell) \longrightarrow \mathscr{Y}\left(n^{-}, n^{+}\right)(k, \ell) \cong \mathscr{Y}
$$

such that $y$ is in the image of the homomorphism $f^{0}=g^{0}$. As $Y^{0}$ is finitely generated as an $R$-module, it follows that there is a finite direct sum as in the statement of the lemma mapping surjectively onto the $Y^{0}$-component.

Similarly, given $y^{+} \in Y^{+}$we find $n^{-}>0$ and $y^{-} \in Y^{-} \otimes_{R_{\leq 0}} R_{\leq n^{-}}$such that $u^{+}\left(y^{+}\right)$is the image of $y^{-}$under the map $z \otimes r \mapsto u^{-}(z) r$. We thus obtain a map of sheaves $\mathscr{O}(0,0) \longrightarrow \mathscr{Y}\left(n^{-}, 0\right)$ by sending the elements 1 to $y^{-}, u^{+}\left(y^{+}\right)$and $y^{+}$, respectively. By twisting, this yields a map of sheaves $\mathscr{O}(k, 0) \longrightarrow \mathscr{Y}$, where $k=-n^{-}<0$, such that $y^{+}$is in the image of the map of +-components. As $Y^{+}$ is finitely generated as an $R_{\geq 0}$-module, it follows that there is a finite direct sum as in the statement of the lemma mapping surjectively onto the $Y^{+}$-component.

This argument works mutatis mutandis for the "-"-component. Combining the three resulting surjections thus constructed yields the requisite map of sheaves.

\section{II.3. SheAF COHOMOLOGY}

Definition II.3.1. Given a sheaf $\mathscr{Y}=\left(Y^{-} \stackrel{u^{-}}{\longrightarrow} Y^{0} \stackrel{u^{+}}{\longrightarrow} Y^{+}\right)$we define its (ČECH) cohomology $R_{0}$-modules by setting $H^{q}\left(\mathbb{P}^{1}, \mathscr{Y}\right)=0$ for $q \geq 2$, and by

$$
H^{0}\left(\mathbb{P}^{1}, \mathscr{Y}\right)=\operatorname{ker}\left(u^{-}-v^{+}\right) \quad \text { and } \quad H^{1}\left(\mathbb{P}^{1}, \mathscr{Y}\right)=\operatorname{coker}\left(u^{-}-u^{+}\right) .
$$

We thus have a canonical exact sequence of $R_{0}$-modules

$$
0 \longrightarrow H^{0}\left(\mathbb{P}^{1}, \mathscr{Y}\right) \longrightarrow Y^{-} \oplus Y^{+} \stackrel{v^{-}-u^{+}}{\longrightarrow} Y^{0} \longrightarrow H^{1}\left(\mathbb{P}^{1}, \mathscr{Y}\right) \longrightarrow 0
$$

which defines the cohomology modules up to canonical isomorphism. Note that $H^{q}\left(\mathbb{P}^{1}, \mathscr{Y}\right)=\lim _{\leftarrow} q \mathscr{Y}$ for all $q \geq 0$, where we consider $\mathscr{Y}$ as a diagram of $R_{0}$-modules. Consequently, a short exact sequence

$$
0 \longrightarrow \mathscr{X} \longrightarrow \mathscr{Y} \longrightarrow \mathscr{Z} \longrightarrow 0
$$


of sheaves yields a not-very-long exact sequence of $R_{0}$-modules

$$
\begin{aligned}
0 \longrightarrow & H^{0}\left(\mathbb{P}^{1}, \mathscr{X}\right) \longrightarrow H^{0}\left(\mathbb{P}^{1}, \mathscr{Y}\right) \longrightarrow H^{0}\left(\mathbb{P}^{1}, \mathscr{Z}\right) \\
& \longrightarrow H^{1}\left(\mathbb{P}^{1}, \mathscr{X}\right) \longrightarrow H^{1}\left(\mathbb{P}^{1}, \mathscr{Y}\right) \longrightarrow H^{1}\left(\mathbb{P}^{1}, \mathscr{Z}\right) \longrightarrow 0
\end{aligned}
$$

We may refer to $H^{0}\left(\mathbb{P}^{1}, \mathscr{Y}\right)$ occasionally as the global sections of $\mathscr{Y}$.

By direct inspection we obtain the following calculation of cohomology modules of the twisting sheaves:

Proposition II.3.4. Let $k, \ell \in \mathbb{Z}$.

(1) If $k+\ell \geq 0$ then $H^{1}\left(\mathbb{P}^{1}, \mathscr{O}(k, \ell)\right)$ is trivial, and there is an isomorphism $H^{0}\left(\mathbb{P}^{1}, \mathscr{O}(k, \ell)\right) \cong \bigoplus_{n=-\ell}^{k} R_{n}$.

(2) If $k+\ell<0$ then $H^{0}\left(\mathbb{P}^{1}, \mathscr{O}(k, \ell)\right)$ is trivial, and there is an isomorphism $H^{1}\left(\mathbb{P}^{1}, \mathscr{O}(k, \ell)\right) \cong \bigoplus_{n=k+1}^{-\ell-1} R_{n}$.

In particular, $H^{0}\left(\mathbb{P}^{1}, \mathscr{O}(k, \ell)\right)$ and $H^{1}\left(\mathbb{P}^{1}, \mathscr{O}(k, \ell)\right)$ are finitely generated projective $R_{0}$-modules.

We now exhibit a phenomenon that does not occur in algebraic geometry: given an integer $n$ and a sheaf $\mathscr{Y}$ there are infinitely many " $n$th twists" of $\mathscr{Y}$, viz., the sheaves $\mathscr{Y}(k, \ell)$ with $k+\ell=n$. This leads to a non-trivial action of $\mathbb{Z}$ on the collection of cohomology modules of $n$th twists of $\mathscr{Y}$ :

Theorem II.3.5. Let $\mathscr{Y}$ be a sheaf. For any $k \in \mathbb{Z}$ there is an isomorphism of $R_{0}$-modules, natural in $\mathscr{Y}$,

$$
H^{q}\left(\mathbb{P}^{1}, \mathscr{Y}(k,-k)\right) \cong H^{q}\left(\mathbb{P}^{1}, \mathscr{Y}\right) \otimes_{R_{0}} R_{k}, \quad q=0,1 .
$$

Proof. Since $R_{k}$ is a projective $R_{0}$-module (Lemma I.2.2), the defining exact sequence $\underline{(\text { II.3.2) }}$ for $H^{q}\left(\mathbb{P}^{1}, \mathscr{Y}\right)$ remains exact after application of $-\otimes_{R_{0}} R_{k}$, resulting in the exact sequence of $R_{0}$-modules

$$
\begin{aligned}
0 \longrightarrow H^{0}\left(\mathbb{P}^{1}, \mathscr{Y}\right) \otimes_{R_{0}} R_{k} & \longrightarrow\left(Y^{-} \otimes_{R_{0}} R_{k}\right) \oplus\left(Y^{+} \otimes_{R_{0}} R_{k}\right) \longrightarrow \\
& \longrightarrow Y^{0} \otimes_{R_{0}} R_{k} \longrightarrow H^{1}\left(\mathbb{P}^{1}, \mathscr{Y}\right) \otimes_{R_{0}} R_{k} \longrightarrow 0 .
\end{aligned}
$$

The middle terms are isomorphic, by Proposition I.2.12, to the modules

$$
\left(Y^{-} \otimes_{R_{\leq 0}} R_{\leq k}\right) \oplus\left(Y^{+} \otimes_{R_{\geq 0}} R_{\geq k}\right) \text { and } Y^{0} \otimes_{R} R,
$$

respectively, so that we actually recover the defining exact sequence for the cohomology of $\mathscr{Y}(k,-k)$. In other words, $H^{q}\left(\mathbb{P}^{1}, \mathscr{Y}(k,-k)\right) \cong H^{q}\left(\mathbb{P}^{1}, \mathscr{Y}\right) \otimes_{R_{0}} R_{k}$ as claimed.

Corollary II.3.6. Let $q \in\{0,1\}$, and suppose $k, \ell, k^{\prime}, \ell^{\prime} \in \mathbb{Z}$ are such that $k+\ell=$ $k^{\prime}+\ell^{\prime}$. For any sheaf $\mathscr{Y}$ we have an equivalence

$$
H^{q}\left(\mathbb{P}^{1}, \mathscr{Y}(k, \ell)\right)=0 \quad \Longleftrightarrow \quad H^{q}\left(\mathbb{P}^{1}, \mathscr{Y}\left(k^{\prime}, \ell^{\prime}\right)\right)=0 .
$$


Proof. In view of the isomorphisms (II.2.5) we may assume, without loss of generality, that $k+\ell=0=k^{\prime}=\ell^{\prime}$ (in effect, we replace $\mathscr{Y}\left(k^{\prime}, \ell^{\prime}\right)$ with $\mathscr{Y}$ in the statement). Then by the previous Theorem $H^{q}\left(\mathbb{P}^{1}, \mathscr{Y}(k, \ell)\right)=0$ if and only if $H^{q}\left(\mathbb{P}^{1}, \mathscr{Y}\right) \otimes_{R_{0}} R_{k}=0$, which happens if and only if $H^{q}\left(\mathbb{P}^{1}, \mathscr{Y}\right)=0$ as $R_{k}$ is an invertible $R_{0}$-bimodule by Lemma I.2.4.

\section{II.4. VECTOR BUNDLES WITH TRIVIAL FIRST COHOMOLOGY}

For $K$-theoretical considerations we will restrict attention to those vector bundles $\mathscr{Y}$ having trivial first cohomology for all their twists $\mathscr{Y}(k, \ell)$ with $k+\ell \geq 0$ : their global sections are finitely generated projective $R_{0}$-modules. We will demonstrate this in Theorem II.4.5, after dispensing with some auxiliary results.

Lemma II.4.1. Let $\mathscr{Y}$ be a sheaf consisting of projective modules with vanishing $H^{1}\left(\mathbb{P}^{1}, \mathscr{Y}\right)$. Then $H^{0}\left(\mathbb{P}^{1}, \mathscr{Y}\right)$ is a projective $R_{0}$-module.

Proof. In view of the hypothesis $H^{1}\left(\mathbb{P}^{1}, \mathscr{Y}\right)=0$, the exact sequence (II.3.2) reduces to a short exact sequence of $R_{0}$-modules

$$
0 \longrightarrow H^{0}\left(\mathbb{P}^{1}, \mathscr{Y}\right) \longrightarrow Y^{-} \oplus Y^{+} \longrightarrow Y^{0} \longrightarrow 0 .
$$

As $R$ is strongly $\mathbb{Z}$-graded, the rings $R_{\leq 0}, R$ and $R_{\geq 0}$ are projective $R_{0}$-modules (Lemma I.2.2). We conclude that projective modules over any of these rings are projective as $R_{0}$-modules; this applies in particular to $Y^{-}, Y^{0}$ and $Y^{+}$. Thus the above short exact sequence splits, and $H^{0}\left(\mathbb{P}^{1}, \mathscr{Y}\right)$ is a direct summand of the projective $R_{0}$-module $Y^{-} \oplus Y^{+}$. Hence $H^{0}\left(\mathbb{P}^{1}, \mathscr{Y}\right)$ is a projective $R_{0}$-module as well.

Lemma II.4.2. Let $\mathscr{Y}$ be a locally finitely generated sheaf. Then there exists $n_{0} \geq 0$ such that $H^{1}\left(\mathbb{P}^{1}, \mathscr{Y}(k, \ell)\right)=0$ for all $k, \ell \in \mathbb{Z}$ with $k+\ell \geq n_{0}$.

Proof. By Lemma II.2.6 there exists an epimorphism $\mathscr{F} \longrightarrow \mathscr{Y}$, where $\mathscr{F}$ is a finite sum of twisting sheaves $\mathscr{O}\left(k_{j}, \ell_{j}\right)$ with $k_{j}, \ell_{j} \leq 0$ and $k_{j}+\ell_{j}<0$. Let $n_{0}$ be the maximum of the positive numbers $-k_{j}-\ell_{j}$ occurring here. We claim that this number has the required property.

To see this, let $k, \ell \in \mathbb{Z}$ with $k+\ell \geq n_{0}$ be given. As twisting is exact we then have an epimorphism $\epsilon: \mathscr{F}(k, \ell) \longrightarrow \mathscr{Y}(k, \ell)$. By (II.2.5) we have

$$
\mathscr{F}(k, \ell)=\bigoplus_{j} \mathscr{O}\left(k_{j}, \ell_{j}\right)(k, \ell) \cong \bigoplus_{j} \mathscr{O}\left(k_{j}+k, \ell_{j}+\ell\right)
$$

with $k_{j}+k+\ell_{j}+\ell \geq 0$, by choice of $n_{0}$, so that $\mathscr{F}(k, \ell)$ has trivial first cohomology by Proposition II.3.4. By (II.3.3) we have an exact sequence

$$
\cdots \longrightarrow \underbrace{H^{1}\left(\mathbb{P}^{1}, \mathscr{F}(k, \ell)\right)}_{=0} \longrightarrow H^{1}\left(\mathbb{P}^{1}, \mathscr{Y}(k, \ell)\right) \longrightarrow 0
$$

proving that $H^{1}\left(\mathbb{P}^{1}, \mathscr{Y}(k, \ell)\right)=0$ as advertised. 
Lemma II.4.3. For every vector bundle $\mathscr{Y}$ there exists a number $n_{0} \geq 0$ such that $H^{0}\left(\mathbb{P}^{1}, \mathscr{Y}(k, \ell)\right)$ is a finitely generated projective $R_{0}$-module for all $k, \ell \in \mathbb{Z}$ with $k+\ell \geq n_{0}$.

Proof. By Lemma II.2.6 there exists a vector bundle $\mathscr{F}=\bigoplus_{j} \mathscr{O}\left(k_{j}, \ell_{j}\right)$ and an epimorphism $\epsilon: \mathscr{F} \longrightarrow \mathscr{Y}$. Then $\mathscr{K}=\operatorname{ker} \epsilon$ is a vector bundle as well, and we choose the integer $n_{0}$, according to Lemma II.4.2, so that both $\mathscr{K}$ and $\mathscr{Y}$ have trivial first cohomology when twisted accordingly. Now suppose that $k+\ell \geq n_{0}$. By Lemma II.4.1 we know that $H^{0}\left(\mathbb{P}^{1}, \mathscr{Y}(k, \ell)\right)$ is a projective $R_{0}$-module. From the short exact sequence

$$
0 \longrightarrow \mathscr{K}(k, \ell) \longrightarrow \mathscr{F}(k, \ell) \longrightarrow \mathscr{Y}(k, \ell) \longrightarrow 0
$$

and (II.3.3) we obtain an exact sequence of $R_{0}$-modules

$$
H^{0}\left(\mathbb{P}^{1}, \mathscr{F}(k, \ell)\right) \stackrel{\alpha}{\longrightarrow} H^{0}\left(\mathbb{P}^{1}, \mathscr{Y}(k, \ell)\right) \longrightarrow H^{1}\left(\mathbb{P}^{1}, \mathscr{K}(k, \ell)\right)=0
$$

so that $\alpha$ is onto. But the domain of $\alpha$ is a finitely generated projective $R_{0}$-module, by Proposition II.3.4, so $H^{0}\left(\mathbb{P}^{1}, \mathscr{Y}(k, \ell)\right)$ is finitely generated.

Definition II.4.4. Let $n \in \mathbb{Z}$. We denote by $\operatorname{Vect}\left(\mathbb{P}^{1}\right)_{n}$ the full subcategory of $\operatorname{Vect}\left(\mathbb{P}^{1}\right)$ consisting of those vector bundles $\mathscr{Y}$ with $H^{1}\left(\mathbb{P}^{1}, \mathscr{Y}(k, \ell)\right)=0$ for all $k, \ell \in \mathbb{Z}$ with $k+\ell \geq n$.

We will see later that the algebraic $K$-theory of the category $\operatorname{Vect}\left(\mathbb{P}^{1}\right)$ is the same as the algebraic $K$-theory of $\operatorname{Vect}\left(\mathbb{P}^{1}\right)_{0}$. The point is that for $\operatorname{Vect}\left(\mathbb{P}^{1}\right)_{0}$ we have the following crucial finiteness result at our disposal:

Theorem II.4.5. For $\mathscr{Y} \in \operatorname{Vect}\left(\mathbb{P}^{1}\right)_{0}$ and $k, \ell \in \mathbb{Z}$ with $k+\ell \geq 0$, the $R_{0}$-module $H^{0}\left(\mathbb{P}^{1}, \mathscr{Y}(k, \ell)\right)$ is finitely generated projective.

Proof. Given integers $k, \ell \in \mathbb{Z}$ with $k+\ell \geq 0$, and given any $j \geq 0$, we obtain from (II.2.3), by tensoring with $\mathscr{Y}$, a short exact sequence of vector bundles

$$
\begin{aligned}
0 \longrightarrow \mathscr{Y}(k, \ell+j) \longrightarrow \mathscr{Y}(k+1, \ell+j) & \oplus \mathscr{Y}(k, \ell+j+1) \\
& \longrightarrow \mathscr{Y}(k+1, \ell+j+1) \longrightarrow 0
\end{aligned}
$$

which by (II.3.3) yields, keeping in mind that $\mathscr{Y}$ is an object in $\operatorname{Vect}\left(\mathbb{P}^{1}\right)_{0}$ by hypothesis, a short exact sequence

$$
\begin{aligned}
& 0 \longrightarrow H^{0}\left(\mathbb{P}^{1}, \mathscr{Y}(k, \ell+j)\right) \\
& \longrightarrow H^{0}\left(\mathbb{P}^{1}, \mathscr{Y}(k+1, \ell+j)\right) \oplus H^{0}\left(\mathbb{P}^{1}, \mathscr{Y}(k, \ell+1+j)\right) \\
& \longrightarrow H^{0}\left(\mathbb{P}^{1}, \mathscr{Y}(k+1, \ell+1+j)\right) \longrightarrow 0 .
\end{aligned}
$$

We choose $n_{0} \gg 0$ sufficiently large according to Lemma II.4.3, applied to the vector bundle $\mathscr{Y}$. We can now use downward induction on $j$, starting with $j=$ $n_{0}-1$. In each step, the second and third term are known to be finitely generated projective $R_{0}$-modules, whence the first term is so as well, for all integers $k, \ell$ 
with $k+\ell \geq 0$. - The last step of the induction is done with $j=0$, proving the claim.

In preparation for our $K$-theoretical deliberations we consider cokernels and pushouts of certain maps of vector bundles.

Lemma II.4.6. Suppose that $\alpha: \mathscr{X} \longrightarrow \mathscr{Y}$ is an injective map of sheaves. Suppose further that $k, \ell \in \mathbb{Z}$ are such that $H^{1}\left(\mathbb{P}^{1}, \mathscr{Y}(k, \ell)\right)=0$. Then the cohomology module $H^{1}\left(\mathbb{P}^{1}, \operatorname{coker}(\alpha)(k, \ell)\right)$ is trivial as well. - In particular, if $\operatorname{coker}(\alpha)$ is a vector bundle, and if $\mathscr{Y}$ is an object of $\operatorname{Vect}\left(\mathbb{P}^{1}\right)_{n}$, then $\operatorname{coker}(\alpha)$ is an object of $\operatorname{Vect}\left(\mathbb{P}^{1}\right)_{n}$.

Proof. As twisting is an exact endofunctor of the category $\mathfrak{Q} \mathfrak{C o h}\left(\mathbb{P}^{1}\right)$, there is a canonical isomorphism $\operatorname{coker}(\alpha)(k, \ell) \cong \operatorname{coker}(\alpha(k, \ell))$ and a resulting short exact sequence of sheaves

$$
0 \longrightarrow \mathscr{X}(k, \ell) \stackrel{\alpha(k, \ell)}{\longrightarrow} \mathscr{Y}(k, \ell) \longrightarrow \operatorname{coker}(\alpha)(k, \ell) \longrightarrow 0 .
$$

The associated exact sequence in cohomology (II.3.3) ends in

$$
H^{1}\left(\mathbb{P}^{1}, \mathscr{Y}(k, \ell)\right) \longrightarrow H^{1}\left(\mathbb{P}^{1}, \operatorname{coker}(\alpha)(k, \ell)\right) \longrightarrow 0
$$

with first term being trivial, by hypothesis on $\mathscr{Y}$. This shows that the second term is trivial as well.

Consider now a commutative square diagram in the category $\mathfrak{Q} \mathfrak{C o h}\left(\mathbb{P}^{1}\right)$ :

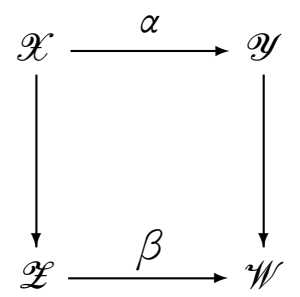

Lemma II.4.8. Suppose that the diagram (II.4.7) is a pushout with $\alpha$ an injective map, and that the sheaves $\mathscr{Z}$ and $\operatorname{coker}(\alpha)$ are vector bundles. Then the map $\beta$ is injective, and both $\mathscr{W}$ and coker $(\beta)$ are vector bundles. If, in addition, $\mathscr{Y}$ and $\mathscr{Z}$ are objects of $\operatorname{Vect}\left(\mathbb{P}^{1}\right)_{n}$ then so is $\mathscr{W}$.

Proof. As the square is a pushout, $\operatorname{coker}(\alpha)$ is isomorphic to $\operatorname{coker}(\beta)$ so that the latter is a vector bundle. For each decoration ? $\in\{-, 0,+\}$ the short exact sequence

$$
0 \longrightarrow X^{?} \stackrel{\alpha^{2}}{\longrightarrow} Y^{?} \longrightarrow \operatorname{coker}\left(\alpha^{?}\right) \longrightarrow 0
$$

splits as the third non-trivial term is a projective module; it follows that $\alpha^{\text {? }}$ is a split injection, whence its pushout $\beta^{\text {? }}$ is a split injection as well. Hence we obtain a split short exact sequence

$$
0 \longrightarrow Z^{?} \stackrel{\beta^{?}}{\longrightarrow} W^{?} \longrightarrow \operatorname{coker}\left(\beta^{?}\right) \longrightarrow 0
$$


with first and third term finitely generated projective (as both $\mathscr{Z}$ and $\operatorname{coker}(\beta)$ are vector bundles). This forces $W^{\text {? }}$ to be finitely generated projective whence $\mathscr{W}$ is a vector bundle.

Now suppose, in addition to the previous hypotheses, that $\mathscr{Y}$ and $\mathscr{Z}$ are objects of $\operatorname{Vect}\left(\mathbb{P}^{1}\right)_{n}$. From the exact sequence of sheaves

$$
0 \longrightarrow \mathscr{Z}(k, \ell) \stackrel{\beta(k, \ell)}{\longrightarrow} \mathscr{W}(k, \ell) \longrightarrow \operatorname{coker}(\beta)(k, \ell) \longrightarrow 0
$$

(note that twisting is exact, and commutes with pushouts as tensor products preserve colimits) we construct an exact sequence snippet in cohomology

$$
H^{1}\left(\mathbb{P}^{1}, \mathscr{Z}(k, \ell)\right) \longrightarrow H^{1}\left(\mathbb{P}^{1}, \mathscr{W}(k, \ell)\right) \longrightarrow H^{1}\left(\mathbb{P}^{1}, \operatorname{coker}(\beta)(k, \ell)\right),
$$

cf. (II.3.3). Since $\mathscr{Z} \in \operatorname{Vect}\left(\mathbb{P}^{1}\right)_{n}$ by hypothesis, and since $\operatorname{coker}(\beta) \cong \operatorname{coker}(\alpha) \in$ $\operatorname{Vect}\left(\mathbb{P}^{1}\right)_{n}$ by Lemma II.4.6, we conclude that the middle term is trivial whenever $k+\ell \geq n$ so that $\mathscr{W} \overline{\in \operatorname{Vect}}\left(\mathbb{P}^{1}\right)_{n}$ as claimed.

\section{Part III. The algebraic $K$-theory of the projective line}

We continue to assume that $R=\bigoplus_{k \in \mathbb{Z}} R_{k}$ is a strongly $\mathbb{Z}$-graded ring.

\section{III.1. Algebraic $K$-THEORY}

A map $f$ of chain complexes of quasi-coherent sheaves will be called a $q$-equivalence, or a quasi-isomorphism, if $f^{\text {? }}$ is a quasi-isomorphism of chain complexes of modules for each decoration ? $\in\{-, 0,+\}$. We let $\mathrm{Ch}^{b} \operatorname{Vect}\left(\mathbb{P}^{1}\right)$ denote the category of bounded chain complexes of vector bundles; similarly, we denote by $\mathrm{Ch}^{b} \operatorname{Vect}\left(\mathbb{P}^{1}\right)_{n}$ the category of bounded chain complexes of vector bundles in $\operatorname{Vect}\left(\mathbb{P}^{1}\right)_{n}$, cf. Definition II.4.4. The categories $\mathrm{Ch}^{b} \operatorname{Vect}\left(\mathbb{P}^{1}\right)$ and $\mathrm{Ch}^{b} \operatorname{Vect}\left(\mathbb{P}^{1}\right)_{n}$ are WALDHAUSEN categories with weak equivalences the quasi-isomorphisms, and cofibrations the injections with cokernel a complex of vector bundles. Existence of the requisite pushouts has been verified above in Lemma II.4.8; note that in the case of $\operatorname{Vect}\left(\mathbb{P}^{1}\right)_{n}$ the cokernel of a cofibration is an object of $\overline{C h}{ }^{b} \operatorname{Vect}\left(\mathbb{P}^{1}\right)_{n}$ again by Lemma II.4.6. - As weak equivalences are defined homologically, they satisfy the saturation and extension axioms. All categories mentioned have a cylinder functor given by the usual mapping cylinder construction which satisfies the cylinder axiom.

Definition III.1.1. The $K$-theory space of the projective line is defined to be

$$
K\left(\mathbb{P}^{1}\right)=\Omega\left|q \mathscr{S}_{\bullet} \mathrm{Ch}^{b} \operatorname{Vect}\left(\mathbb{P}^{1}\right)\right|,
$$

where " $q$ " stands for the category of quasi-isomorphisms.

By Lemma II.4.2, the category $\mathrm{Ch}^{b} \operatorname{Vect}\left(\mathbb{P}^{1}\right)$ is the increasing union of the categories $\mathrm{Ch}^{b} \operatorname{Vect}\left(\mathbb{P}^{1}\right)_{n}, n \geq 0$. This filtration allows us to restrict attention to the category $\operatorname{Vect}\left(\mathbb{P}^{1}\right)_{0}$ as far as algebraic $K$-theory is concerned. Before we formally prove this result, let us mention that twisting $\mathscr{Y} \mapsto \mathscr{Y}(k, \ell)$ is an additively exact 
auto-equivalence that preserves quasi-isomorphisms, cofibrations and pushouts, with the functor $\mathscr{Z} \mapsto \mathscr{Z}(-k,-\ell))$ being an inverse. We also introduce new notation for the twisting functor:

$$
\vartheta_{k, \ell}: \operatorname{Vect}\left(\mathbb{P}^{1}\right) \longrightarrow \operatorname{Vect}\left(\mathbb{P}^{1}\right), \quad \mathscr{Y} \mapsto \mathscr{Y}(k, \ell) .
$$

Lemma III.1.3. For all $n \in \mathbb{N}$ the inclusion $\operatorname{Vect}\left(\mathbb{P}^{1}\right)_{n} \subseteq \operatorname{Vect}\left(\mathbb{P}^{1}\right)_{n+1}$ induces a bomotopy equivalence $K\left(\operatorname{Vect}\left(\mathbb{P}^{1}\right)_{n}\right) \simeq K\left(\operatorname{Vect}\left(\mathbb{P}^{1}\right)_{n+1}\right)$.

Proof. Let $k, \ell \in \mathbb{Z}$. If $k+\ell \geq 0$ the functor $\vartheta_{k, \ell}$ restricts to a functor

$$
\operatorname{Vect}\left(\mathbb{P}^{1}\right)_{n} \longrightarrow \operatorname{Vect}\left(\mathbb{P}^{1}\right)_{n}
$$

which we denote by the same symbol $\vartheta_{k, \ell}$. In case $k+\ell \geq 1$ we may consider this as a functor $\vartheta_{k, \ell}: \operatorname{Vect}\left(\mathbb{P}^{1}\right)_{n+1} \longrightarrow \operatorname{Vect}\left(\mathbb{P}^{1}\right)_{n}$. From the exact sequence of twisting sheaves (II.2.3) we conclude that there is a short exact sequence of functors

$$
0 \longrightarrow \mathrm{id} \longrightarrow \vartheta_{1,0} \oplus \vartheta_{0,1} \longrightarrow \vartheta_{1,1} \longrightarrow 0
$$

(we have used $\vartheta_{0,0} \cong$ id here). By the additivity theorem ([Wal85], Proposition 1.3.2 (4) and Theorem 1.4.2) that means that, on the level of $K$-groups, the identity map is the difference of the maps induced by $\vartheta_{1,0} \oplus \vartheta_{0,1}$ and $\vartheta_{1,1}$, both as endofunctors of $\operatorname{Vect}\left(\mathbb{P}^{1}\right)_{n}$ and of $\operatorname{Vect}\left(\mathbb{P}^{1}\right)_{n+1}$. This in turn implies that the difference of the induced maps of the functors $\vartheta_{1,0} \oplus \vartheta_{0,1}$ and $\vartheta_{1,1}$, considered as functors $\operatorname{Vect}\left(\mathbb{P}^{1}\right)_{n+1} \longrightarrow \operatorname{Vect}\left(\mathbb{P}^{1}\right)_{n}$, is both left and right inverse to the map in $K$-theory induced by the inclusion functor $\operatorname{Vect}\left(\mathbb{P}^{1}\right)_{n} \stackrel{\subseteq}{\longrightarrow} \operatorname{Vect}\left(\mathbb{P}^{1}\right)_{n+1}$.

Corollary III.1.4. The inclusion $\operatorname{Vect}\left(\mathbb{P}^{1}\right)_{0} \subseteq \operatorname{Vect}\left(\mathbb{P}^{1}\right)$ induces a homotopy equivalence $\Omega\left|q \mathscr{S}_{\bullet} \mathrm{Ch}^{b} \operatorname{Vect}\left(\mathbb{P}^{1}\right)_{0}\right| \stackrel{\simeq}{\longrightarrow} K\left(\mathbb{P}^{1}\right)$.

\section{III.2. CANONICAL SHEAVES AND GLOBAL SECTIONS}

We write $\mathrm{Ch}^{b} \mathbf{M}\left(R_{0}\right)$ for the category of bounded chain complexes of (right) $R_{0}$-modules. Its full subcategory of bounded chain complexes of finitely generated projective $R_{0}$-modules is denoted $\mathrm{Ch}^{b} \mathrm{P}\left(R_{0}\right)$. We consider the latter as a WALDHAUSEN category with weak equivalences the homotopy equivalences (or equivalently, the quasi-isomorphisms), and cofibrations the injections with levelwise projective cokernel. The associated $K$-theory space is denoted by $K\left(R_{0}\right)=$ $\Omega\left|h \mathscr{S}_{\bullet} \mathrm{Ch}^{\mathrm{b}} \mathbf{P}\left(R_{0}\right)\right|$; its homotopy groups are the QUILLEN $K$-groups of the $\operatorname{ring} R_{0}$.

We define functors, the canonical sheaves functors,

$$
\Psi_{k, \ell}: \mathrm{Ch}^{b} \mathrm{P}\left(R_{0}\right) \longrightarrow \mathrm{Ch}^{b} \operatorname{Vect}\left(\mathbb{P}^{1}\right), \quad C \mapsto C \otimes \mathscr{O}(k, \ell)
$$

where $C \otimes \mathscr{O}(k, \ell)$ denotes the bounded complex of sheaves

$$
C \otimes_{R_{0}} R_{\leq k} \longrightarrow C \otimes_{R_{0}} R \longleftarrow C \otimes_{R_{0}} R_{\geq-\ell} .
$$


By Lemma I.2.2 the constituents of $\mathscr{O}(k, \ell)$ are invertible bimodules over their respective ground rings. Hence $C \otimes \mathscr{O}(k, \ell)$ consists of finitely generated projective modules, and the functor $\Psi_{k, \ell}$ is an exact functor between WALDHAUSEN categories.

\section{Lemma III.2.1. There are isomorphisms of ABELian groups}

$$
\operatorname{hom}\left(\Psi_{0,0}(C), \mathscr{Y}\right) \cong \operatorname{hom}\left(C, H^{0}\left(\mathbb{P}^{1}, \mathscr{Y}\right)\right)
$$

which are natural in $C$ and in $\mathscr{Y}$. In other words, the functor $\Psi_{0,0}$ is left adjoint to the global sections functor $H^{0}\left(\mathbb{P}^{1},-\right)$.

Proof. Since $H^{0}\left(\mathbb{P}^{1}, \mathscr{Y}\right)=\lim _{\leftarrow} \mathscr{Y}$, linear maps $C \longrightarrow H^{0}\left(\mathbb{P}^{1}, \mathscr{Y}\right)$ correspond bijectively to commutative diagrams of the type

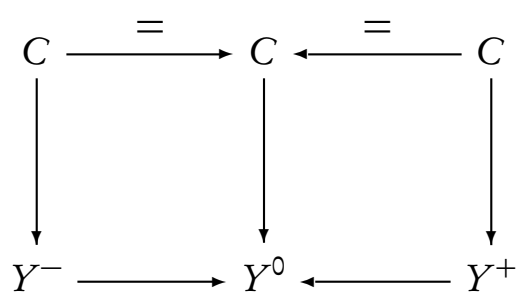

with $R_{0}$-linear vertical maps. By the usual extension of scalars construction, such diagrams correspond bijectively to diagrams of the type

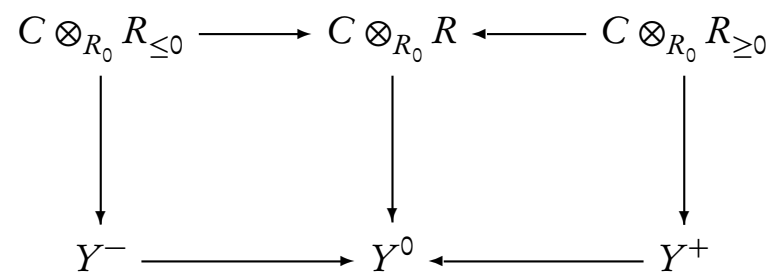

which are maps $\Psi_{0,0}(C) \longrightarrow \mathscr{Y}$.

Lemma III.2.2. For $C \in \mathrm{Ch}^{b} \mathbf{P}\left(R_{0}\right)$ we have natural isomorphisms

$$
H^{q}\left(\mathbb{P}^{1}, \Psi_{k, \ell}(C)\right) \cong C \otimes_{R_{0}} H^{q}\left(\mathbb{P}^{1}, \mathscr{O}(k, \ell)\right), \quad q=0,1,
$$

where sheaf cohomology is computed in each chain level separately. In particular,

(1) $H^{0}\left(\mathbb{P}^{1}, \Psi_{0,0}(C)\right) \cong C$,

(2) $H^{0}\left(\mathbb{P}^{1}, \Psi_{k, \ell}(C)\right)=0$ if $k+\ell \leq-1$,

(3) $H^{1}\left(\mathbb{P}^{1}, \Psi_{k, \ell}(C)\right)=0$ if $k+\ell \geq-1$.

Proof. For $\mathscr{Y}=\mathscr{O}(k, \ell)$ the canonical sequence (II.3.2) becomes an exact sequence of $R_{0}-R_{0}$-bimodule chain complexes

$$
\begin{aligned}
& 0 \longrightarrow H^{0}\left(\mathbb{P}^{1}, \mathscr{O}(k, \ell)\right) \longrightarrow R_{\leq k} \oplus R_{\geq-\ell} \\
& \longrightarrow R \longrightarrow H^{1}\left(\mathbb{P}^{1}, \mathscr{O}(k, \ell)\right) \longrightarrow 0 .
\end{aligned}
$$

Tensoring this with $C$ over $R_{0}$ yields another exact sequence as $C$ consists of projective $R_{0}$-modules; the result is in fact the canonical sequence (II.3.2) for the 
chain complex of vector bundles $\mathscr{Y}=\Psi_{k, \ell}(C)$, which thus has $q$ th cohomology isomorphic to $C \otimes_{R_{0}} H^{q}\left(\mathbb{P}^{1}, \mathscr{O}(k, \ell)\right)$. - The assertions (1), (2) and (3) follow from what we just proved and the calculations of Proposition II.3.4:

(1) $H^{0}\left(\mathbb{P}^{1}, \mathscr{O}(0,0)\right) \cong R_{0}$;

(2) $H^{0}\left(\mathbb{P}^{1}, \mathscr{O}(k, \ell)\right)=0$ if $k+\ell \leq-1$;

(3) $H^{1}\left(\mathbb{P}^{1}, \mathscr{O}(k, \ell)\right)=0$ if $k+\ell \geq-1$.

Corollary III.2.3. Let $i, j \in \mathbb{Z}$. If $i+j \geq-1$, the functor $\Psi_{i, j}$ factors through the category $\mathrm{Ch}^{b} \operatorname{Vect}\left(\mathbb{P}^{1}\right)_{0}$.

Proof. We need to verify that $H^{1}\left(\mathbb{P}^{1}, \Psi_{i, j}(C)(k, \ell)\right)=0$ if $k+\ell \geq 0$, for any chain complex $C \in \mathrm{Ch}^{b} \mathbf{P}\left(R_{0}\right)$. By $\underline{(I I .2 .5)}$ we have an isomorphism $\Psi_{i, j}(C)(k, \ell) \cong$ $\Psi_{i+k, j+\ell}(C)$ and thus, by the previous Lemma, the required vanishing result.

For $i+j \geq-1$ the functor $\Psi_{i, j}$ associates a bounded complex of vector bundles in $\operatorname{Vect}\left(\mathbb{P}^{1}\right)_{0}$ with a bounded complex of $R_{0}$-modules. We will need a functor going the other direction; we use the global sections functor $\Gamma=H^{0}\left(\mathbb{P}^{1},-\right)$. Explicitly,

$$
\Gamma: \mathrm{Ch}^{b} \operatorname{Vect}\left(\mathbb{P}^{1}\right)_{0} \longrightarrow \mathrm{Ch}^{b} \mathrm{P}\left(R_{0}\right), \quad \mathscr{Y} \mapsto H^{0}\left(\mathbb{P}^{1}, \mathscr{Y}\right) .
$$

This is well defined by virtue of Theorem II.4.5: the complex $H^{0}\left(\mathbb{P}^{1}, \mathscr{Y}\right)$ consists of finitely generated projective $R_{0}$-modules for $\mathscr{Y} \in \mathrm{Ch}^{b} \operatorname{Vect}\left(\mathbb{P}^{1}\right)_{0}$. Note also that $\Gamma$ is an (additive) exact functor, i.e., $\Gamma$ maps short exact sequences in $\mathrm{Ch}^{b} \operatorname{Vect}\left(\mathbb{P}^{1}\right)_{0}$ to short exact sequences in $\mathrm{Ch}^{b} \mathrm{P}\left(R_{0}\right)$; this follows immediately from the fact that twisting is an additive exact functor, and from (II.3.3) as all first cohomology modules vanish. Consequently, the functor $\Gamma$ automatically preserves all pushouts that exist in $\operatorname{Vect}\left(\mathbb{P}^{1}\right)_{0}$.

Lemma III.2.4. The functor $\Gamma: \mathrm{Ch}^{b} \operatorname{Vect}\left(\mathbb{P}^{1}\right)_{0} \longrightarrow \mathrm{Ch}^{b} \mathbf{P}\left(R_{0}\right)$ is an exact functor of WALDHAUSEN categories.

Proof. The non-trivial points to check are the following:

(1) If $f: \mathscr{Y} \longrightarrow \mathscr{Z}$ is a cofibration, then $\Gamma(f)$ is a cofibration.

(2) If $f: \mathscr{Y} \longrightarrow \mathscr{Z}$ is a weak equivalence, then $\Gamma(f)$ is a weak equivalence.

To prove (1) recall that coker $(f)$ is, by definition of cofibrations, a complex of vector bundles, by Lemma II.4.6 in fact a complex of vector bundles in $\operatorname{Vect}\left(\mathbb{P}^{1}\right)_{0}$. As $\Gamma$ is additively exact we obtain a short exact sequence

$$
0 \longrightarrow \Gamma(\mathscr{Y}) \stackrel{\Gamma(f)}{\longrightarrow} \Gamma(\mathscr{Z}) \longrightarrow \Gamma(\operatorname{coker} f) \longrightarrow 0
$$

of $R_{0}$-modules. But this sequence consists of chain complexes of projective modules, so $\Gamma(f)$ is a levelwise split injection with levelwise projective cokernel, that is, is a cofibration in $\mathrm{Ch}^{b} \mathbf{P}\left(R_{0}\right)$. 
As for (2), since we are working with vector bundles in $\operatorname{Vect}\left(\mathbb{P}^{1}\right)_{0}$ the cohomology sequence (II.3.3) reduces to a short exact sequence

$$
0 \longrightarrow \Gamma(\mathscr{Y}) \longrightarrow Y^{-} \oplus Y^{+} \stackrel{u^{-}-u^{+}}{\longrightarrow} Y^{0} \longrightarrow 0
$$

so that $\Gamma(\mathscr{Y})$ is quasi-isomorphic to the "homotopy fibre" $\mathrm{H}\left(u^{-}-u^{+}\right)$of the map $u^{-}-u^{+}$, i.e., to the totalisation of $Y^{-} \oplus Y^{+} \stackrel{u^{-}-u^{+}}{\longrightarrow} Y^{0}$ considered as a double complex. But this latter construction is known to be invariant under quasiisomorphisms, so that a weak equivalence $f: \mathscr{Y} \stackrel{\sim}{\longrightarrow} \mathscr{Z}$ yields a square diagram

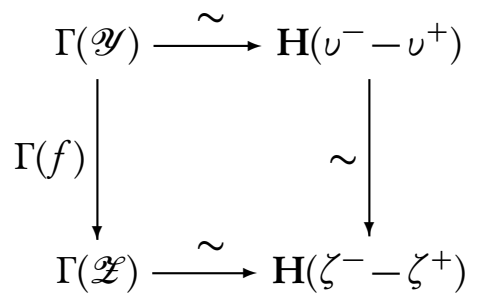

whence $\Gamma(f)$ is a quasi-isomorphism as required.

\section{III.3. WEAK EQUIVALENCES OF COMPLEXES OF SHEAVES}

As usual in the context of WALDHAUSEN $K$-theory we need various notions of weak equivalences on the category concerned. As defined before, a quasi-isomorphism in $\mathrm{Ch}^{b} \operatorname{Vect}\left(\mathbb{P}^{1}\right)_{0}$ is map $f: \mathscr{Y} \longrightarrow \mathscr{Z}$ such that its components $f$ ?, for $? \in\{-, 0,+\}$, are quasi-isomorphisms of chain complexes of modules.

Lemma III.3.1. Let $f: \mathscr{Y} \longrightarrow \mathscr{Z}$ be a map in $\mathrm{Ch}^{b} \operatorname{Vect}\left(\mathbb{P}^{1}\right)$, and let $k_{0}, \ell_{0}, n \in \mathbb{Z}$ with $k_{0}+\ell_{0}=n$ be fixed integers. Each of the following statements implies the others:

(1) The map $\Gamma \circ \vartheta_{k_{0}, \ell_{0}}(f)$ is a quasi-isomorphism in $\mathrm{Ch}^{b} \mathbf{M}\left(R_{0}\right)$.

(2) There exist $k, \ell \in \mathbb{Z}$ with $k+\ell=n$ such that the map $\Gamma \circ \vartheta_{k, \ell}(f)$ is a quasiisomorphism in $\mathrm{Ch}^{b} \mathbf{M}\left(R_{0}\right)$.

(3) For all $k, \ell \in \mathbb{Z}$ with $k+\ell=n$, the map $\Gamma \circ \vartheta_{k, \ell}(f)$ is a quasi-isomorphism in $\mathrm{Ch}^{b} \mathbf{M}\left(R_{0}\right)$.

Proof. First, we may replace the map $f$ by $\vartheta_{n, 0}(f)$ and thus reduce to the case $n=0$, using the isomorphisms (II.2.5). So assume $n=0$. For $k+\ell=0$ there is a natural isomorphism $\Gamma \circ \vartheta_{k, \ell}\left(\overline{f) \cong \Gamma}(f) \otimes_{R_{0}} R_{k}\right.$, by Theorem II.3.5. As $R_{k}$ is a

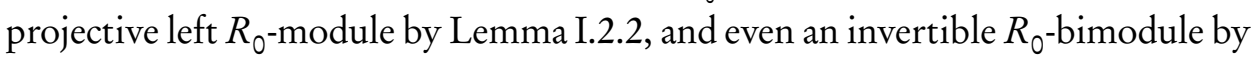
Lemma I.2.4, we conclude that $\Gamma(f)$ is a quasi-isomorphism if and only if $\Gamma \circ \vartheta_{k, \ell}$ is. The result follows.

Definition III.3.2. A map $f: \mathscr{Y} \longrightarrow \mathscr{Z}$ in $\operatorname{Ch}^{b} \operatorname{Vect}\left(\mathbb{P}^{1}\right)_{0}$ is a qo-equivalence if it satisfies one (hence all) of the three equivalent conditions listed in Lemma III.3.1 for $n=0$. We call $f$ a $q_{1}$-equivalence if it satisfies one (hence all) of the equivalent conditions listed in Lemma III.3.1 for $n=0$ and for $n=1$. 
Equipped with $q_{\text {? }}$-equivalences, ? $=0,1$, and the previous notion of cofibrations the category $\mathrm{Ch}^{b} \operatorname{Vect}\left(\mathbb{P}^{1}\right)_{0}$ is a WALDHAUSEN category satisfying the saturation and extension axioms. The gluing lemma follows from the fact that twisting preserves pushouts, and from the fact that $\Gamma$ preserves pushouts and cofibrations by Lemma III.2.4, as the category $\mathrm{Ch}^{b} \mathbf{P}\left(R_{0}\right)$ is known to satisfy the gluing lemma. The usual mapping cylinder construction provides a cylinder functor which satisfies the cylinder axiom.

\section{III.4. ACYCLIC COMPLEXES OF VECTOR BUNDLES}

We turn our attention to a characterisation of acyclic complexes of vector bundles with trivial higher cohomology: If global sections of "zeroth" and "first" twists are trivial, the complex is quasi-isomorphic to the zero complex. The precise result is formulated as follows:

Proposition III.4.1. Let $\mathscr{Y} \in \mathrm{Ch}^{b} \operatorname{Vect}\left(\mathbb{P}^{1}\right)_{0}$ be a complex of vector bundles such that the canonical map $\zeta: \mathscr{Y} \longrightarrow 0$ is a q-equivalence, cf. Definition III.3.2. Then $\zeta$ is a q-equivalence, that is, $\mathscr{Y}$ is quasi-isomorphic to the zero complex.

Proof. The exact sequence of sheaves

$$
0 \longrightarrow \mathscr{O}(0,0) \longrightarrow \mathscr{O}(1,0) \longrightarrow \mathscr{O}(1,0) / \mathscr{O}(0,0) \longrightarrow 0
$$

yields, by pointwise tensor product with the complex of vector bundles $\mathscr{Y}$, an exact sequence of complexes of sheaves

$$
0 \longrightarrow \mathscr{Y} \stackrel{\alpha}{\longrightarrow} \mathscr{Y}(1,0) \longrightarrow \mathscr{Z} \longrightarrow 0
$$

with $\mathscr{Z}=\operatorname{coker}(\alpha)$ satisfying $Z^{0}=0$ and $Z^{+}=0$. Explicitly, we obtain the diagram of chain complexes of $R_{0}$-modules with exact columns depicted in Fig. 1. The $R_{\leq 0}$-module structure of $R_{1}$ in the third non-trivial row is determined by

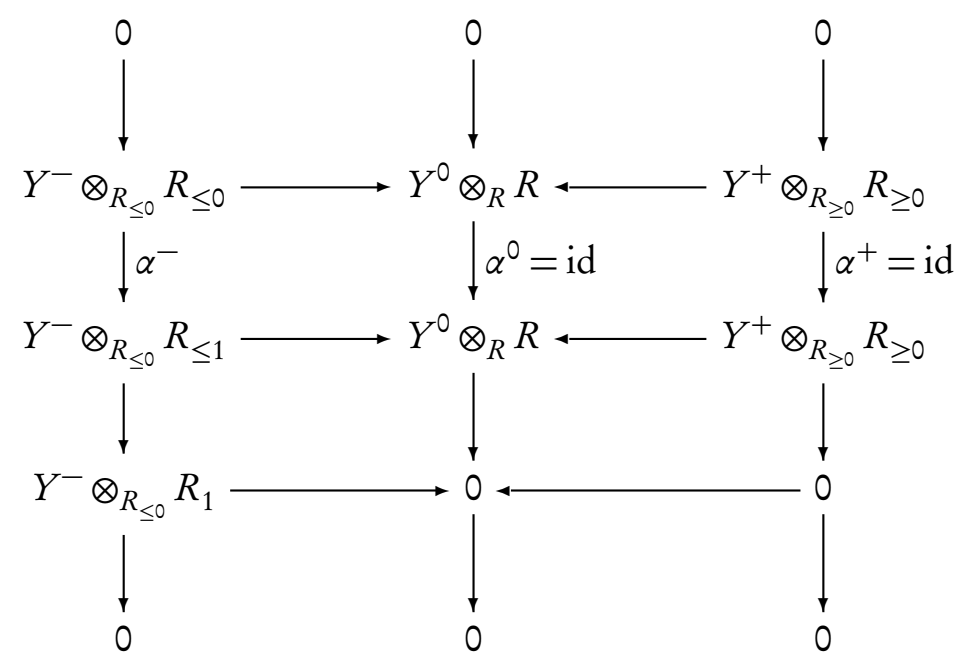

FIGURE 1. Diagram used in proof of Proposition III.4.1 
saying that all elements of strictly negative degree act on $R_{1}$ as multiplication by 0 . - The first horizontal slice is $\mathscr{Y}(0,0) \cong \mathscr{Y}$, while the second is the vector bundle $\vartheta_{1,0}(\mathscr{Y})=\mathscr{Y}(1,0)$. The first slice has levelwise trivial $\lim ^{1}$; this is true by definition of $\operatorname{Vect}\left(\mathbb{P}^{1}\right)_{0}$ and by the equality $\lim _{\leftarrow}^{1}=H^{1}\left(\mathbb{P}^{1},-\right)$. As a consequence, applying the functor $\lim$ results in a short exact sequence of $R_{0}$-modules

$$
0 \longrightarrow \Gamma(\mathscr{Y}) \longrightarrow \Gamma \circ \vartheta_{1,0}(\mathscr{Y}) \longrightarrow Y^{-} \otimes_{R_{\leq 0}} R_{1} \longrightarrow 0
$$

with contractible first and second terms, by our hypothesis on $\mathscr{Y}$. So the complex $Y^{-} \otimes_{R_{\leq 0}} R_{1}=\operatorname{coker}\left(\alpha^{-}\right)$has trivial homology. But $\alpha^{-}$is a map of bounded chain complexes of projective $R_{\leq 0}$-modules, whence $\alpha^{-}$is actually a homotopy equivalence. By tensoring over $R_{\leq 0}$ with $R_{\leq p}$ from the right, and using the $R_{\leq 0}$-bimodule isomorphism $R_{\leq p^{\prime}} \otimes_{R_{\leq 0}} R_{\leq p} \cong R_{\leq p^{\prime}+p}$, we conclude that the map

$$
\alpha_{p}^{-}: Y^{-} \otimes_{R_{\leq 0}} R_{\leq p} \longrightarrow Y^{-} \otimes_{R_{\leq 0}} R_{\leq p+1}
$$

is a homotopy equivalence for all $p \geq 0$. But then the map $\mu^{-}$from $Y^{-} \cong$ $Y^{-} \otimes_{R_{\leq 0}} R_{\leq 0}$ to the colimit $W^{-}$of the sequence

$$
Y^{-} \otimes_{R_{\leq 0}} R_{\leq 0} \stackrel{\alpha_{0}^{-}}{\longrightarrow} Y^{-} \otimes_{R_{\leq 0}} R_{\leq 1} \stackrel{\alpha_{1}^{-}}{\longrightarrow} Y^{-} \otimes_{R_{\leq 0}} R_{\leq 2} \stackrel{\alpha_{2}^{-}}{\longrightarrow} \cdots
$$

is a quasi-isomorphism since homology commutes with filtered colimits. As this colimit is precisely $W^{-}=Y^{-} \otimes_{R_{\leq 0}} \bigcup_{p \geq 0} R_{\leq p}=Y^{-} \otimes_{R_{\leq 0}} R$, and as $\mathscr{Y}$ is a complex of vector bundles, the composite map

$$
Y^{-} \stackrel{\mu^{-}}{\sim} W^{-}=Y^{-} \otimes_{R_{\leq 0}} R \underset{\sharp}{\stackrel{u_{\sharp}^{-}}{\cong}} Y^{0}
$$

is a quasi-isomorphism. That is, the structure map $Y^{-} \stackrel{u^{-}}{\longrightarrow} Y^{0}$ of $\mathscr{Y}$ is a quasiisomorphism. - A similar argument, employing $\mathscr{Y}(0,1)$ in place of $\mathscr{Y}(1,0)$, shows that the structure map $Y^{0} \longleftarrow Y^{+}$is a quasi-isomorphism as well.

We have shown that $\mathscr{Y}$ is quasi-isomorphic, as a diagram of chain complexes of projective $R_{0}$-modules, to the constant diagram

$$
\mathscr{C}_{Y}=\left(Y^{0} \stackrel{\mathrm{id}}{\longrightarrow} Y^{0} \stackrel{\mathrm{id}}{\longrightarrow} Y^{0}\right),
$$

which has levelwise trivial $\lim _{\leftarrow}^{1}$. It follows that

$$
Y^{0} \underset{(\mathrm{i})}{\cong} \lim _{\leftarrow} \mathscr{C}_{Y} \underset{(\mathrm{ii})}{\simeq} \lim \mathscr{Y}=\Gamma(\mathscr{Y}) \simeq 0,
$$

that is, $Y^{0}$ is acyclic. (The isomorphism (i) is the result of direct computation, while for the quasi-isomorphism (ii) we can use an argument similar to the one used in Lemma III.2.4, part (2) of the proof.) As $Y^{-}$and $Y^{+}$are quasi-isomorphic to $Y^{0}$, as shown above, this proves $\mathscr{Y} \simeq 0$ as required. 


\section{III.5. THE SPLITTING RESULT}

Theorem III.5.1. Suppose that $R=\bigoplus_{k \in \mathbb{Z}} R_{k}$ is a strongly $\mathbb{Z}$-graded ring. There is a homotopy equivalence of $K$-theory spaces

$$
K\left(R_{0}\right) \times K\left(R_{0}\right) \longrightarrow K\left(\mathbb{P}^{1}\right)
$$

induced by the functor

$$
\begin{aligned}
\Psi_{-1,0}+\Psi_{0,0}: \mathrm{Ch}^{b} \mathbf{P}\left(R_{0}\right) \times \mathrm{Ch}^{b} \mathbf{P}\left(R_{0}\right) & \longrightarrow \mathrm{Ch}^{b} \operatorname{Vect}\left(\mathbb{P}^{1}\right) \\
(C, D) & \mapsto \Psi_{-1,0}(C) \oplus \Psi_{0,0}(D) .
\end{aligned}
$$

Proof. By Corollary III.2.3 the functor $\Psi_{0,0}+\Psi_{-1,0}$ factors through the category $\mathrm{Ch}^{b} \operatorname{Vect}\left(\mathbb{P}^{1}\right)_{0}$ which, in view of Corollary III.1.4, has the same $K$-theory (with respect to $q$-equivalences) as the category $\mathrm{Ch}^{b} \operatorname{Vect}\left(\mathbb{P}^{1}\right)$. It is thus enough to show that the functor

$$
\begin{aligned}
\Psi_{-1,0}+\Psi_{0,0}: \operatorname{Ch}^{b} \mathbf{P}\left(R_{0}\right) \times \operatorname{Ch}^{b} \mathbf{P}\left(R_{0}\right) & \longrightarrow \operatorname{Ch}^{b} \operatorname{Vect}\left(\mathbb{P}^{1}\right)_{0} \\
(C, D) & \mapsto \Psi_{-1,0}(C) \oplus \Psi_{0,0}(D)
\end{aligned}
$$

induces a homotopy equivalence on $K$-theory spaces (with respect to quasi-isomorphisms as weak equivalence in both source and target).

Claim III.5.2. Let $q_{0}$ denote the category of $q_{0}$-equivalences in $\operatorname{Vect}\left(\mathbb{P}^{1}\right)_{0}$, see Definition III.3.2. There is a fibration sequence

$$
\left|q \mathscr{S}_{\bullet} \mathrm{Ch}^{b} \operatorname{Vect}\left(\mathbb{P}^{1}\right)_{0}^{q_{0}}\right| \longrightarrow\left|q \mathscr{S}_{\bullet} \mathrm{Ch}^{b} \operatorname{Vect}\left(\mathbb{P}^{1}\right)_{0}\right| \stackrel{\Gamma}{\longrightarrow}\left|h \mathscr{S}_{\bullet} \mathrm{Ch}^{b} \mathbf{P}\left(R_{0}\right)\right|
$$

which has a section up to homotopy induced by the functor $\Psi_{0,0}$.

Indeed, every quasi-isomorphism in $\mathrm{Ch}^{b} \operatorname{Vect}\left(\mathbb{P}^{1}\right)_{0}$ is a $q_{0}$-equivalence. The machinery of WALDHAUSEN $K$-theory [Wal85, Theorem 1.6.4] thus provides us with a fibration sequence

$$
\left|q \mathscr{S}_{\bullet} \operatorname{Ch}^{b} \operatorname{Vect}\left(\mathbb{P}^{1}\right)_{0}^{q_{0}}\right| \longrightarrow\left|q \mathscr{S}_{\bullet} \operatorname{Ch}^{b} \operatorname{Vect}\left(\mathbb{P}^{1}\right)_{0}\right| \longrightarrow\left|q_{0} \mathscr{S}_{\bullet} \mathrm{Ch}^{b} \operatorname{Vect}\left(\mathbb{P}^{1}\right)_{0}\right|
$$

The base is homotopy equivalent to $\left|h \mathscr{S}_{\bullet} \mathrm{Ch}^{b} \mathrm{P}\left(R_{0}\right)\right|$, via the functors $\Gamma$ and $\Psi_{0,0}$; in fact, $\Gamma \circ \Psi_{0,0} \cong \mathrm{id}$ by Lemma III.2.2, and the adjunction counit $\epsilon: \Psi_{0,0} \circ \mathrm{\Gamma} \mathrm{id}$ (associated with the adjunction of Lemma III.2.1) is a $q_{0}$-equivalence as application of $\Gamma$ yields, up to canonical isomorphism, the identity transformation $\mathrm{id}=\Gamma(\epsilon): \Gamma \circ \Psi_{0,0} \circ \Gamma \longrightarrow \Gamma$. From $\Gamma \circ \Psi_{0,0} \cong \mathrm{id}$ we also conclude that $\Psi_{0,0}$ induces a section up to homotopy of the fibration sequence (III.5.3). This proves the claim.

Since the fibration sequence (III.5.3) has a section up to homotopy, the associated long exact sequence of homotopy groups (which are ABELian groups in this 
case) decomposes into split short exact sequences

$$
\begin{aligned}
0 \longrightarrow \pi_{k}\left|q \mathscr{S}_{\bullet} \operatorname{Ch}^{b} \operatorname{Vect}\left(\mathbb{P}^{1}\right)_{0}^{q_{0}}\right| & \longrightarrow \pi_{k}\left|q \mathscr{S}_{\bullet} \mathrm{Ch}^{b} \operatorname{Vect}\left(\mathbb{P}^{1}\right)_{0}\right| \\
& \stackrel{\Gamma}{\longrightarrow} \pi_{k}\left|h \mathscr{S}_{\bullet} \operatorname{Ch}^{b} \mathbf{P}\left(R_{0}\right)\right| \longrightarrow 0
\end{aligned}
$$

so that there are group isomorphisms

$$
\pi_{k}\left|q \mathscr{S}_{\bullet} \mathrm{Ch}^{b} \operatorname{Vect}\left(\mathbb{P}^{1}\right)_{0}^{q_{0}}\right| \oplus \pi_{k}\left|h \mathscr{S}_{\bullet} \mathrm{Ch}^{b} \mathbf{P}\left(R_{0}\right)\right| \stackrel{\lambda}{\longrightarrow} \pi_{k}\left|q \mathscr{S}_{\bullet} \mathrm{Ch}^{b} \operatorname{Vect}\left(\mathbb{P}^{1}\right)_{0}\right|
$$

with $\lambda$ induced by the functor $(\mathscr{Y}, \mathscr{Z}) \mapsto \mathscr{Y} \oplus \Psi_{0,0}(\mathscr{Z})$. It follows that this functor yields a homotopy equivalence

$$
\left|q \mathscr{S} \cdot \mathrm{Ch}^{b} \operatorname{Vect}\left(\mathbb{P}^{1}\right)_{0}^{q_{0}}\right| \times\left|h \mathscr{S}_{\bullet} \mathrm{Ch}^{b} \mathbf{P}\left(R_{0}\right)\right| \stackrel{\left(\text { incl } \Psi_{0,0}\right)}{\simeq}\left|q \mathscr{S}_{\bullet} \mathrm{Ch}^{b} \operatorname{Vect}\left(\mathbb{P}^{1}\right)_{0}\right| \cdot(\text { III.5.4) }
$$

We proceed by analysing the fibre term of the fibration sequence (III.5.3).

Claim III.5.5. There is another fibration sequence, similar to the one above,

$$
\left|q \mathscr{S}_{\bullet} \mathrm{Ch}^{b} \operatorname{Vect}\left(\mathbb{P}^{1}\right)_{0}^{q}\right| \longrightarrow\left|q \mathscr{S}_{\bullet} \mathrm{Ch}^{b} \operatorname{Vect}\left(\mathbb{P}^{1}\right)_{0}^{q_{0}}\right| \stackrel{\Gamma \circ \vartheta_{1,0}}{\longrightarrow}\left|h \mathscr{S}_{\bullet} \mathrm{Ch}^{b} \mathbf{P}\left(R_{0}\right)\right|
$$

which has a section up to homotopy induced by the functor $\Psi_{-1,0}$.

Indeed, let $q_{1}$ denote the category of $q_{1}$-equivalences in $\operatorname{Vect}\left(\mathbb{P}^{1}\right)_{0}^{q_{0}}$, see Definition III.3.2. The general $K$-theory machinery provides a fibration sequence

$$
\left|q \mathscr{S}_{\bullet} \mathrm{Ch}^{b} \operatorname{Vect}\left(\mathbb{P}^{1}\right)_{0}^{q_{1}}\right| \longrightarrow \mid q \mathscr{S}_{\bullet} \mathrm{Ch}^{b} \operatorname{Vect}\left(\mathbb { P } ^ { 1 } | _ { 0 } ^ { q _ { 0 } } | \longrightarrow | q _ { 1 } \mathscr { S } _ { \bullet } \mathrm { Ch } ^ { b } \operatorname { V e c t } \left(\left.\mathbb{P}^{1}\right|_{0} ^{q_{0}} \mid\right.\right. \text {. }
$$

Again, the base is homotopy equivalent to $\left|h \mathscr{S}_{\bullet} \mathrm{Ch}^{b} \mathbf{P}\left(R_{0}\right)\right|$ via the functors $\Gamma \circ \vartheta_{1,0}$ and $\Psi_{-1,0}$; note that $\Gamma \circ \Psi_{-1,0}=0$ by Lemma III.2.2 so that $\Psi_{-1,0}$ takes indeed values in $\operatorname{Vect}\left(\mathbb{P}^{1}\right)_{0}^{q_{0}}$. We have $\left(\Gamma \circ \vartheta_{1,0}\right) \circ \Psi_{-1,0}=\Gamma \circ \overline{=}_{0,0} \cong \mathrm{id}$, and the adjunction counit

$$
\Psi_{-1,0} \circ\left(\Gamma \circ \vartheta_{1,0}\right) \longrightarrow \mathrm{id}
$$

is transformed, by application of $\Gamma \circ \vartheta_{1,0}$, into the identity map. So there results a fibration sequence

$$
\left|q \mathscr{S}_{\bullet} \mathrm{Ch}^{b} \operatorname{Vect}\left(\mathbb{P}^{1}\right)_{0}^{q_{1}}\right| \longrightarrow\left|q \mathscr{S}_{\bullet} \mathrm{Ch}^{b} \operatorname{Vect}\left(\mathbb{P}^{1}\right)_{0}^{q_{0}}\right| \stackrel{\Gamma \circ \vartheta_{1,0}}{\longrightarrow}\left|h \mathscr{S}_{\bullet} \mathrm{Ch}^{b} \mathbf{P}\left(R_{0}\right)\right|,
$$

and the isomorphism $\left(\Gamma \circ \vartheta_{1,0}\right) \circ \Psi_{-1,0} \cong$ id shows that $\Psi_{-1,0}$ provides a section up to homotopy. The category $\mathrm{Ch}^{b} \operatorname{Vect}\left(\mathbb{P}^{1}\right)_{0}^{q_{1}}$ in the fibre term consists of those complexes of vector bundles $\mathscr{Y} \in \operatorname{Vect}\left(\mathbb{P}^{1}\right)_{0}$ such that $\mathscr{Y} \longrightarrow 0$ is a $q_{1}$-equivalence, that is, by Proposition III.4.1, such that $\mathscr{Y} \simeq 0$. Thus we have an equality of categories $\mathrm{Ch}^{b} \operatorname{Vect}\left(\mathbb{P}^{1}\right)_{0}^{q_{1}}=\mathrm{Ch}^{b} \operatorname{Vect}\left(\mathbb{P}^{1}\right)_{0}^{q}$, and the fibration sequence (III.5.7) is actually the desired sequence (III.5.6). Its fibre term is contractible. This means that $\Gamma \circ \vartheta_{1,0}$ induces a homotopy equivalence; consequently, the section up to homotopy, induced by $\Psi_{-1,0}$, is a homotopy equivalence as well. 
Combining this homotopy equivalence with (III.5.4) yields a homotopy equivalence

$$
\left|h \mathscr{S}_{\bullet} \mathrm{Ch}^{b} \mathbf{P}\left(R_{0}\right)\right| \times\left|h \mathscr{S}_{\bullet} \mathrm{Ch}^{b} \mathbf{P}\left(R_{0}\right)\right| \stackrel{\left(\Psi_{-1,0} \Psi_{0,0}\right)}{\simeq}\left|q \mathscr{S}_{\bullet} \mathrm{Ch}^{b} \operatorname{Vect}\left(\mathbb{P}^{1}\right)_{0}\right|
$$

Looping once and combining with the homotopy equivalence

$$
\Omega\left|h \mathscr{S}_{\bullet} \mathrm{Ch}^{b} \operatorname{Vect}\left(\mathbb{P}^{1}\right)_{0}\right| \stackrel{\simeq}{\longrightarrow} \Omega\left|h \mathscr{S}_{\bullet} \operatorname{Ch}^{b} \operatorname{Vect}\left(\mathbb{P}^{1}\right)\right|=K\left(\mathbb{P}^{1}\right)
$$

from Corollary III.1.4 gives the advertised splitting result.

\section{REFERENCES}

[Bas68] Hyman Bass. Algebraic K-theory. W. A. Benjamin, Inc., New York-Amsterdam, 1968.

[Bou98] Nicolas Bourbaki. Algebra I. Chapters 1-3. Elements of Mathematics (Berlin). Springer-Verlag, Berlin, 1998. Translated from the French, Reprint of the 1989 English translation.

[Dad80] Everett C. Dade. Group-graded rings and modules. Math. Z., 174(3):241-262, 1980.

[Gra88] Daniel R. Grayson. The $K$-theory of semilinear endomorphisms. J. Algebra, 113(2):358-372, 1988.

[Haz13] R. Hazrat. The graded structure of Leavitt path algebras. Israel J. Math., 195(2):833-895, 2013.

[HS17] Thomas Hüttemann and Luke Steers. Finite domination and Novikov homology over strongly $\mathbb{Z}$-graded rings. Israel J. Math., 221(2):661-685, 2017.

[Hüt20] Thomas Hüttemann. The "fundamental theorem" for the algebraic $K$-theory of strongly $\mathbb{Z}$-graded rings, 2020.

[Qui73] Daniel Quillen. Higher algebraic K-theory. I. In Algebraic K-theory, I: Higher K-theories (Proc. Conf., Battelle Memorial Inst., Seattle, Wash., 1972), pages 85-147. Lecture Notes in Math., Vol. 341. Springer, Berlin, 1973.

[Wal85] Friedhelm Waldhausen. Algebraic $K$-theory of spaces. In Algebraic and geometric topology (New Brunswick, N.J., 1983), volume 1126 of Lecture Notes in Math., pages 318-419. Springer, Berlin, 1985.

[Yao95] Dongyuan Yao. A note on the $K$-theory of twisted projective lines and twisted Laurent polynomial rings. J. Algebra, 173(2):424-435, 1995.

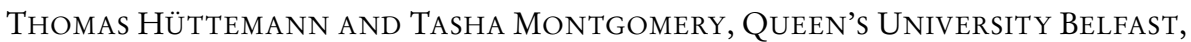
SCHOOl of MATHEMATICS AND Physics, Mathematical SCIENCES ResearCH

CENTRE, BELFAST BT7 1NN, UK

E-mail address: t.huettemann@qub.ac.uk

$U R L:$ https://t-huettemann.github.io/

$U R L:$ https ://pure.qub.ac.uk/en/persons/thomas-huettemann 